\title{
Nonparametric Signed-rank Control Charts with Variable Sampling Intervals
}

\author{
M.L.I. Coelho ${ }^{a}$, M.A. Graham ${ }^{a, *}$ and S. Chakraborti ${ }^{a, b}$
}

\begin{abstract}
Variable sampling intervals (VSI) charts have been proposed in the literature on normal theory (parametric) control charts and are known to provide performance enhancements. In the VSI setting, the time between monitored samples is allowed to vary depending on (the outcome) what is observed in the current sample. Nonparametric (distribution-free) control charts have recently come to play an important role in the field of Statistical Process Control (SPC) and monitoring. In this paper a nonparametric Shewhart-type VSI control chart is considered for detecting changes in a specified location parameter. The proposed chart is based on the Wilcoxon signed-rank statistic and is called the VSI signed-rank chart. The VSI signed-rank chart is compared with an existing fixed sampling interval (FSI) signed-rank chart, the parametric VSI $\bar{X}$-chart and the nonparametric VSI sign chart. Results show that the VSI signed-rank chart often performs favourably and should be used.
\end{abstract}

Keywords: Fixed Sampling Interval, Shewhart control chart, Signed-rank, Statistical Process Control, Variable Sampling Interval

\section{Introduction}

Control charts are commonly used chart in Statistical Process Control (SPC) to determine whether a process is in-control (IC), or to bring an out-of-control (OOC) process to IC and to monitor a process to make sure that it stays IC. The usual practice in control charting is to monitor a process by taking samples at a fixed time interval, say every hour (see, for example, Reynolds et al. ${ }^{1}$ ). This is commonly referred to as a control chart with a fixed sampling interval (FSI). In recent times, researchers have explored the idea of sampling and monitoring the process at different time points with variable sampling interval (VSI) control charts. The reasons for using VSI charts are to minimise the time it takes to detect a shift in the process, as well as to optimize the number of samples that are needed to be taken to detect a shift. In some instances this can greatly reduce the cost. Reynolds et al. ${ }^{1}$ was the first to consider an $\bar{X}$-chart with variable sampling intervals (VSI). Since then, many authors, such as

\footnotetext{
${ }^{a}$ Department of Statistics, University of Pretoria, Pretoria, South Africa

${ }^{b}$ Department of Information Systems, Statistics and Management Science, University of Alabama, Tuscaloosa, AL 35487, U.S.A.

*Correspondence to: MA Graham, Tel: +27 12420 6637, Postal address: Natural Sciences Building 4-7, Groenkloof camps, Leyds Street, Pretoria, South Africa, 0002, marien.graham@up.ac.za
} 
Reynolds et al. ${ }^{2}$, Rendtel ${ }^{3}$, Saccucci et al. ${ }^{4}$, Amin and Widmaier ${ }^{5}$, Reynolds and Arnold ${ }^{6}$, Jensen et al. ${ }^{7}$, Epprecht et al. ${ }^{8}$, Ou et al. ${ }^{9}$, Zhang et al. ${ }^{10}$ have considered the VSI idea in the context of different control charting methods. For the VSI charts the key idea is to monitor a process by allowing taking samples at different time intervals (points). These time intervals are decided upon on where the charting statistic plots most recently on the control chart. If it plots closer to the centre line $(C L)$ and below the upper warning limit $(U W L)$ and above the lower warning limit $(L W L)$ then a longer time interval is used. This is done as it is reasonable to take more time, because there is no evidence that the process is OOC or is moving towards being OOC. On the other hand, if the current charting statistic plots on or above the upper warning limit (UWL) or on or below the lower warning limit (LWL), but still falls within the control limits, then a shorter time interval is used before the next sample is taken, as it is expected that the process could be moving towards being OOC. It has become common practice that two different time intervals are used, see Reynolds et al. ${ }^{1}$. These two time intervals are defined as units of the original FSI time interval. An example is shown in Figure 1.

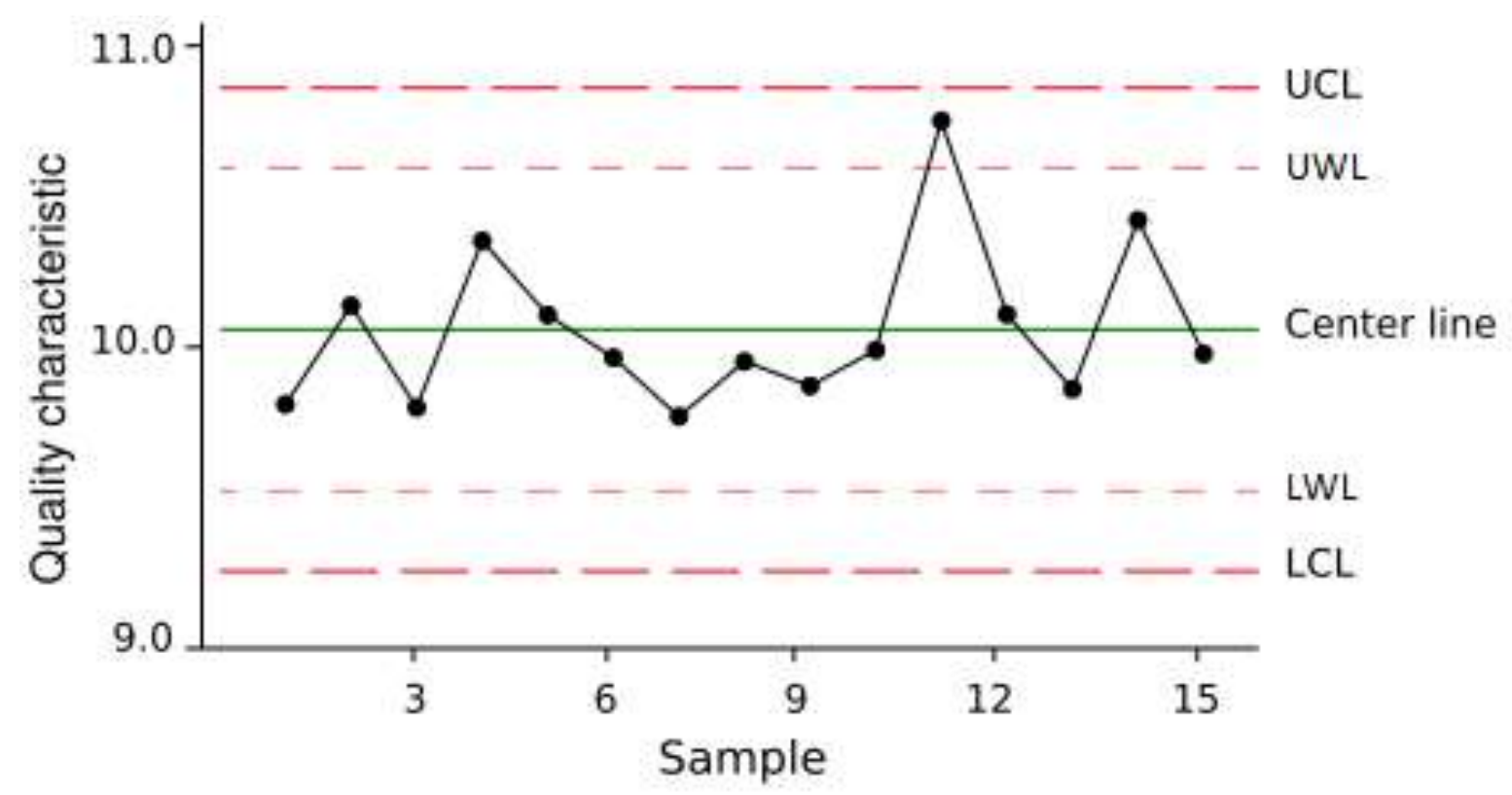

Figure 1. A typical Shewhart-type VSI control chart

Reynolds and Arnold ${ }^{6}$, Reynolds ${ }^{11}$, Runger and Pignatiello ${ }^{12}$ and Reynolds ${ }^{13}$ all provide proof that the dual interval method is the best. Reynolds and Arnold ${ }^{6}$ provide justification for the one-sided Shewhart VSI $\bar{X}$-chart and Reynolds ${ }^{11}$ extends this idea to the two-sided Shewhart VSI $\bar{X}$-chart as well as to charts that can be modelled using the Markov chain 
approach. Runger and Pignatiello ${ }^{12}$ provide proof of the dual interval method for one-sided as well as two-sided charts. However, all of these charts are parametric charts, that is, they assume that the underlying process distribution is normal or approximately normal. Such charts are known to suffer from in-control non-robustness. This means that if such a chart is applied to a process that is non-normal, there is a much higher false alarm rate $(F A R)$, that is too many false alarms are observed relative to what is nominally expected for that chart, since the control limits are inappropriate.

Nonparametric charts are not designed with any assumption of a specific underlying distribution in mind. This means that they are robust over a much larger class of underlying distributions of various shapes and the IC run-length distribution of the control chart remains the same over that class of distributions. This IC robustness is a great asset in practice as it allows the user the flexibility of not assuming normality or some other parametric distribution. There has been a lot of interest in nonparametric statistical process control (NSPC) charts recently. The interested reader is referred to Chakraborti et al. ${ }^{14}$, Chakraborti and Graham ${ }^{15}$ and Chakraborti et al. ${ }^{16}$. However, in the NSPC literature there has not been much work done on VSI control charts. Amin and Widmaier ${ }^{5}$ proposed a Shewhart-type VSI control chart based on the sign statistic. According to our knowledge, no other nonparametric VSI control chart has been proposed to date.

In this paper, we propose a nonparametric VSI control chart based on the signed-rank statistic since it is known (see Gibbons and Chakraborti ${ }^{17}$ ) that the signed-rank test is a more powerful test than the sign test. The assumption for the signed-rank test is that the process distribution is continuous and symmetric. Hence, the proposed VSI signed-rank chart can be used to monitor the location of an unknown continuous but symmetric process distribution.

\section{Adaptive control charts}

In the literature on parametric control charts, adaptive charts have been proposed which are either variable sampling sizes (VSS), or variable sampling intervals (VSI), or possibly a combination of both (VSSI). The adaptive charts have been shown to be faster at detecting shifts than the traditional fixed sampling size (FSS) and fixed sampling interval (FSI) control charts. First we provide some background.

Reynolds et al. ${ }^{1}$ were the first to consider the VSI $\bar{X}$-chart and compare it to the FSI $\bar{X}$ chart. This was done under the assumption of normality. They proposed that random samples of size $n$ are taken at each sampling (time) point, with $\boldsymbol{X}_{i}=\left(X_{i 1}, X_{i 2}, \ldots, X_{i n}\right)$ representing the sample taken at the $i^{\text {th }}$ time point. For each sample, the mean, $\bar{X}_{i}$, is computed, plotted on 
the control chart with limits given by: $C L=\mu_{0}$ and $L C L / U C L=\mu_{0} \pm r(\sigma / \sqrt{n})$ and compared to these control limits. Reynolds et al. ${ }^{1}$ took $r$ to be equal to 3 as in a 3 -sigma chart. For the VSI chart, the time interval between two consecutive samples $\boldsymbol{X}_{i}$ and $\boldsymbol{X}_{i+1}$ is chosen by looking at the value of $\bar{X}_{i}$. They assumed that samples are taken at a finite number of intervals of lengths $d_{1}, d_{2}, \ldots, d_{\eta}$. However, they only used two different time interval lengths. These lengths were chosen so that $l_{1}<d_{i}<l_{2}$ where $l_{1}$ is the minimum possible interval length $\left(l_{1}>0\right)$ and $l_{2}$ is the maximum possible length. In general, the region between the lower and the upper control limits is partitioned into $\eta$ regions, $I_{1}, I_{2}, \ldots, I_{\eta}$ where $d_{j}, j=1,2, \ldots, \eta$, denotes the time unit until sampling the next sample when the charting statistic plots in $I_{j}$. In case only two interval lengths are chosen, there are two regions, $I_{1}$ and $I_{2}$, defined as

$$
I_{1}=\left(\mu_{0}-r \sigma / \sqrt{n}, \mu_{0}-r^{\prime} \sigma / \sqrt{n}\right] \cup\left[\mu_{0}+r^{\prime} \sigma / \sqrt{n}, \mu_{0}+r \sigma / \sqrt{n}\right)
$$

and

$$
I_{2}=\left(\mu_{0}-r^{\prime} \sigma / \sqrt{n}, \mu_{0}+r^{\prime} \sigma / \sqrt{n}\right)
$$

where $r$ is frequently taken to be three and $0<r^{\prime}<3$.

When there is a shift in the process mean, the charting statistics are more likely to plot close to the control limits, i.e. in region $I_{1}$ and, accordingly, sampling will occur at a greater frequency, which in turn will reduce the time it takes before the shift in the mean is detected. Reynolds et al. ${ }^{1}$ also suggested that, for a VSI chart, it would be beneficial if the mean was to be plotted against time instead of the sample number, as this will indicate which time interval length was used for which sample. This is fairly easy to construct when $d_{2}=c d_{1}$ and $c$ is some small positive constant. The choices of $d_{1}$ and $d_{2}$ depends on the specific discipline, i.e. it depends on how long the person is willing to wait to go without sampling.

The most common performance measure for a FSI chart is the average run-length $(A R L)$, which is the average number of points to be plotted before a signal is observed. However, VSI chart properties are measured in terms of the expected length of time it takes before a signal is observed. The length of time it takes before a signal is observed should be long when the process is IC and short when there is a shift in the process. For the FSI scheme it is easy to convert the $A R L$ into an expected time to signal, since the time interval is constant. The expected time to signal is given by the length of the time interval multiplied by the $A R L$. With a VSI chart, however, the time to signal is not a constant multiple of the number of samples needed to signal. The time to signal on a VSI chart depends on both the time to 
signal and the number of samples to signal. Thus in case of a VSI chart, two popular performance measures used are the average number of samples to signal (ANSS) and the average time to signal (ATS), which are both functions of the process mean.

If the control limits were to remain fixed for the VSI chart then the probability that $\bar{X}$ will fall on or outside the control limits remains the same as for the FSI chart.

If $p_{j}=\operatorname{Pr}\left(d(\bar{X})=d_{j}\right)=\operatorname{Pr}\left(\bar{X} \in I_{j}\right)$, then the ATS is given by

$$
A T S=\sum_{j=1}^{\eta} d_{j} p_{j} / \alpha(1-\alpha)
$$

with $\alpha=$ the probability that $\bar{X}$ falls on or outside the control limits, where $d_{j}=$ the time interval in units of the FSI time interval and $I_{j}=$ the region into which the sample falls.

The ATS, however, assumes that the shift occurs at the start of the process monitoring. In reality, the shift can occur and will most likely occur somewhere during the course of the process monitoring. The process mean starts off at $\mu=\mu_{0}$ when the process is IC and shifts to $\mu=\mu_{1}$ (goes OOC) with $\mu_{0} \neq \mu_{1}$ at some random time in the future. In such a situation the time from the shift until the time at which the shift was detected is of interest and the ATS is not an appropriate measure. For such a case Reynolds et al. ${ }^{1}$ proposed the performance measure called the adjusted ATS (denoted AATS).

If $p_{0 j}=P\left(\bar{X} \in I_{j} \mid \mu=\mu_{0}\right), p_{1 j}=P\left(\bar{X} \in I_{j} \mid \mu=\mu_{1}\right)$ and $\alpha_{1}$ denotes probability of a signal in the OOC case $\left(\mu=\mu_{1}\right)$, then the AATS when the shift is to $\mu=\mu_{1}$ is given by

$$
A A T S=\frac{\sum_{j=1}^{2} d_{j}^{2} p_{0 j}}{2 \sum_{j=1}^{2} d_{j} p_{0 j}}+\frac{1}{\alpha_{1}} \sum_{j=1}^{2} d_{j} p_{1 j}
$$

Recall that the AATS gives the expected time to signal from the time when the process mean changed from $\mu_{0}$ to $\mu_{1}$. In the comparison of FSI charts with VSI charts, both charts are designed to have the same $A T S$ and $A R L$ when the process is IC, i.e. both have the same sampling rate and the same $F A R$ over time. Then it is reasonable to compare the values of the AATS functions for various values of $\mu_{1}$ to determine which chart requires fewer time units on an average to give a signal when there has been a mean shift.

\section{Nonparametric adaptive control charts}

The focus of this paper is on the nonparametric VSI control charts. To the best of our knowledge, the only available nonparametric adaptive control chart is the VSI sign control chart proposed by Amin and Widmaier ${ }^{5}$. 


\subsection{The FSI and the VSI sign charts}

First we describe the FSI sign chart. The charting statistic for the sign chart is $T_{i}$, which is the number of sample observations which exceed the in-control median $\mu_{0}$. Amin and Widmaier ${ }^{5}$ give the calculation of $T_{i}$ as

$$
T_{i}=\left\{\sum_{j=1}^{n} \operatorname{sign}\left(X_{i j}-\mu_{0}\right)+n\right\} / 2, \quad i=1,2, \ldots
$$

where $T_{i}$ follows a Binomial distribution with parameters $n$ and $p=P\left(X_{i j}>\mu_{0}\right)$, with $p=0.5$ in the IC case and $\mu_{0}$ is the in-control median of the process.

The nonparametric FSI sign chart control limits are given by $U C L=c$ and $L C L=$ $n-c$. The chart signals if $T_{i}>c$ in the one-sided case for detecting increases in $p$, to signal if $T_{i}<n-c$ in the one-sided case for detecting decreases in $p$, and to signal if $T_{i}>c$ or if $T_{i}<n-c$ in the two-sided case ${ }^{\dagger}$. The value of $c$, which is an integer, can be chosen such that a sign chart with a fixed sample size has a specified (nominal) in-control ARL. In the twosided case for detecting an upper or a lower shift, the chart constant $c$ is chosen such that

$$
1-\alpha_{0}=\sum_{j=n-c}^{c}\left(\begin{array}{l}
n \\
j
\end{array}\right)(0.5)^{n}
$$

where $1-\alpha_{0}$ is the specified $F A R$. The in-control $A R L$ (denoted $\left.A R L_{0}\right)$ is given by

$$
A R L_{0}=\frac{2^{n}}{1-\sum_{j=n-c}^{c}\left(\begin{array}{c}
n \\
j
\end{array}\right)}=\frac{2^{n}}{\sum_{j=0}^{n-c-1}\left(\begin{array}{c}
n \\
j
\end{array}\right)+\sum_{j=c+1}^{n}\left(\begin{array}{c}
n \\
j
\end{array}\right)}=\frac{2^{n-1}}{\sum_{j=c+1}^{n}\left(\begin{array}{c}
n \\
j
\end{array}\right)} .
$$

Amin and Widmaier ${ }^{5}$ also used the AATS, see Equation (2.4), as a measure of performance as suggested by Reynolds et al. ${ }^{1}$, since that can be used when the shift occurs at any point during process monitoring and it does not assume that the shift was present at the start of the process, which is what the ATS assumes.

\footnotetext{
${ }^{\dagger}$ Typically a control chart signals when the charting statistic plots on or above the UCL or on or below the LCL. Amin and Widmaier ${ }^{5}$ however specified the signal to occur only when the charting statistic plots above the UCL or below the LCL. For this reason this method was also followed in Section 3.2, where we proposed a VSI signed-rank control chart.
} 


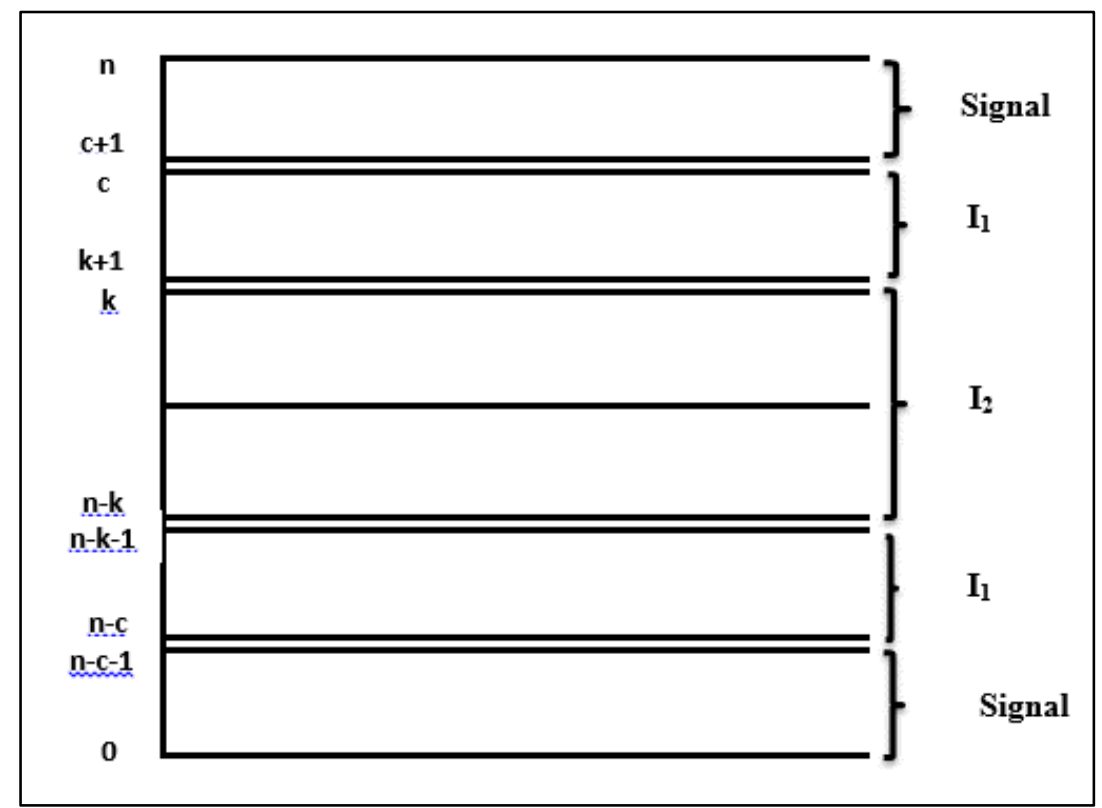

Figure 2. Regions of the VSI sign control chart

Next we describe the VSI sign chart. In a two-sided VSI sign chart, the values $(n-c, \ldots, c)$ correspond to the IC case. Figure 2 shows the partitioning of the range of discrete values $(n-c, \ldots, c)$ into two regions, $I_{1}$ and $I_{2}$, where $I_{2}=\{n-k, \ldots, k)$ and $I_{1}=\{n-c, \ldots, n-k-1\} \cup\{k+l, \ldots, c\}$. The signalling region corresponds to the values $\{0, \ldots, n-c-1\} \cup\{c+l, \ldots, n\}$. We can write the probability of using sampling interval $d_{2}$ as

$$
p_{2}=P\left(T_{i} \in I_{2}\right)=P\left(n-k \leq T_{i} \leq k\right)=\sum_{j=n-k}^{k}\left(\begin{array}{c}
n \\
j
\end{array}\right) p^{j}(1-p)^{n-j}
$$

and the probability of using sampling interval $d_{1}$ as

$$
\begin{aligned}
p_{1}=P\left(T_{i} \in I_{1}\right)=P & \left(n-c \leq T_{i}<n-k\right)+P\left(k<T_{i} \leq c\right) \\
& =\sum_{j=n-c}^{n-k-1}\left(\begin{array}{l}
n \\
j
\end{array}\right) p^{j}(1-p)^{n-j}+\sum_{j=k+1}^{c}\left(\begin{array}{l}
n \\
j
\end{array}\right) p^{j}(1-p)^{n-j} .
\end{aligned}
$$

When the process is IC, $p_{2}$ and $p_{1}$ simplify to

$$
p_{02}=P\left(T_{i} \in I_{2} \mid \mu=\mu_{0}\right)=\sum_{j=n-k}^{k}\left(\begin{array}{c}
n \\
j
\end{array}\right) 0.5^{n}
$$

and

$p_{01}=P\left(T_{i} \in I_{1} \mid \mu=\mu_{0}\right)=\sum_{j=n-c}^{n-k-1}\left(\begin{array}{c}n \\ j\end{array}\right) 0.5^{n}+\sum_{j=k+1}^{c}\left(\begin{array}{c}n \\ j\end{array}\right) 0.5^{n}=2 \sum_{j=k+1}^{c}\left(\begin{array}{c}n \\ j\end{array}\right) 0.5^{n}$, respectively. 
Hence the probability of a signal is given by

$$
\alpha=\sum_{j=0}^{n-c-1}\left(\begin{array}{c}
n \\
j
\end{array}\right) p^{j}(1-p)^{n-j}+\sum_{j=c+1}^{n}\left(\begin{array}{c}
n \\
j
\end{array}\right) p^{j}(1-p)^{n-j} .
$$

This also simplifies in the IC case to

$$
\alpha_{0}=\sum_{j=0}^{n-c-1}\left(\begin{array}{c}
n \\
j
\end{array}\right) 0.5^{n}+\sum_{j=c+1}^{n}\left(\begin{array}{c}
n \\
j
\end{array}\right) 0.5^{n} .
$$

\section{Nonparametric VSI signed-rank chart for location}

The signed-rank test is a nonparametric test that can be used to test hypotheses on or construct confidence intervals (see Gibbons and Chakraborti ${ }^{17}$ ) for the median of any symmetric continuous population distribution. A FSI signed-rank chart was proposed by Bakir $^{18}$. The charting statistic proposed by Bakir ${ }^{18}$ is linearly related to the well-known Wilcoxon signed-rank statistic $W^{+}$. The chart proposed by Bakir ${ }^{18}$ is symmetrical about zero, which makes the $C L=0$ in all cases and this is easier for setting up the chart, however we want to compare the VSI signed-rank chart to the VSI sign chart proposed by Amin and Widmaier ${ }^{5}$. In this work we use the well-known Wilcoxon signed-rank statistic as our charting statistic, as the existing VSI sign chart is not symmetrical about zero, and therefore the $C L$ will not be equal to zero and it will differ for each case.

We propose the Shewhart-type VSI signed-rank control chart to monitor the process location $\theta$, which is the median. We assume that the process is IC when $\theta=\theta_{0}$, a known or a specified value. We assume that the observations from the process output are independent and come from a symmetrical continuous distribution. Under the assumptions of continuity and symmetry, the median is the same as the mean and the Wilcoxon signed-rank statistic is distribution-free. We start with the setting up of the FSI signed-rank chart as this is also the foundation of the VSI chart.

\subsection{The FSI and the VSI signed-rank charts}

At each sampling instance, $t$, regardless of the time interval for sampling, take a random sample of size $n>1$, i.e. $\left(x_{t 1}, x_{t 2}, \ldots, x_{t n}\right)$ for $t=1,2, \ldots$, from a continuous cdf $F$, with a median $\theta_{0}$. We assume that $F$ is symmetric about $\theta_{0}$. The Wilcoxon signed-rank statistic $W^{+}$(the sum of absolute ranks of positive differences) is defined as

$$
W^{+}=\sum_{j=1}^{n} I\left(x_{t j}-\theta_{0}\right) R_{t j}^{+}, \quad t=1,2, \ldots
$$

where

$$
I(x)=\left\{\begin{array}{l}
1 \text { if } x>0 \\
0 \text { if } x \leq 0
\end{array}\right.
$$




$$
R_{t j}^{+}=1+\sum_{i=1}^{n} I\left(\left|x_{t i}-\theta_{0}\right|<\left|x_{t j}-\theta_{0}\right|\right)
$$

where $I(u)=\left\{\begin{array}{l}1 \text { if } u \text { is true } \\ 0 \text { if } u \text { is false }\end{array}\right.$ for $R_{i j}^{+}$. Then $R_{i j}^{+}$is referred to as the within-group absolute rank of the deviations $\left|x_{t i}-\theta_{0}\right|$. The charting statistic $W^{+\prime}$ s for each sample is plotted on the vertical axis against the subgroup number on the horizontal axis along with the associated control limits. Next we consider setting up the FSI and VSI control limits.

\subsection{Setting up of the control limits}

Similar to Bakir ${ }^{18}$, the control limits are chosen in such a way that the chart has a specified $A R L_{0}$, which is equal to the reciprocal of the $F A R$. This makes the comparison of charts under shifts easier as they start off on an equal footing. For a two-sided control chart the $L C L$ and the $U C L$ are chosen to attain a certain $A R L_{0}$. Since the distributions are assumed to be symmetrical it makes sense to have symmetrical control limits. If we have a sample size $n$, let $U C L=c$ where $c$ is some discreet value chosen to obtain a specified $A R L_{0}$. Following this we let $L C L=\frac{n(n+1)}{2}-c$. For a one-sided chart, only one control limit is needed. With nonparametric charts, where the charting statistic has a discrete IC distribution, the usual practice is to deem a process OOC when a charting statistic plots on or outside the control limit(s). However, Amin and Widmaier ${ }^{5}$ in their VSI sign paper defined a signal to be observed when the charting statistic plots outside the control limits and we adopt the same method here in order to have a fair comparison. Therefore a two-sided FSI signed-rank control chart signals when $W^{+}<L C L$ or $W^{+}>U C L$. Similarly, an upper one-sided FSI signed-rank chart signals when $W^{+}>U C L$ and a lower one-sided FSI signed-rank chart signals when $W^{+}<L C L$. From this the probabilities of the charts signaling are given as

$$
\begin{gathered}
\alpha^{+}=\operatorname{Pr}\left(W^{+}>U C L\right) \text { for upper one-sided } \\
\alpha^{-}=\operatorname{Pr}\left(W^{+}<L C L\right) \text { for lower one-sided } \\
\alpha=\operatorname{Pr}\left(W^{+}>U C L\right)+\operatorname{Pr}\left(W^{+}<L C L\right) \quad \text { for two-sided }
\end{gathered}
$$

The run-length follows a geometric distribution with the parameters $\alpha^{+}, \alpha^{-}$and $\alpha$ for the upper one-sided, lower one-sided and two-sided charts, respectively. The ARLs for the upper one-sided, lower one-sided and two-sided charts are then given by

$$
\begin{aligned}
& A R L^{+}=1 / \alpha^{+} \\
& A R L^{-}=1 / \alpha^{-}
\end{aligned}
$$




$$
A R L=1 / \alpha
$$

respectively. The $A R L$ is a measure used to test the performance of FSI charts in Phase II. The chart is typically designed to have a specified $A R L_{0}$ and then the performance is tested by comparing the OOC $A R L$ (denoted $A R L_{\delta}$ ) values when a shift has occurred in the process. The $A R L_{0}$ is usually taken to be large and the $A R L_{\delta}$ should be small, so as to detect the shift fast. The reader is referred to Human and $\operatorname{Graham}^{19}$ for detailed discussions on the ARL.

\subsection{The VSI signed-rank control chart}

The setup of the VSI control chart starts off in the same manner as the set-up of the FSI control chart. However for a VSI chart the $A R L$ is not a good measure of performance as it does not take into account the time it takes before a shift in the process has been detected, it only takes into account the average number of samples until a signal is observed. When using VSI charts the time between samples varies which affects the time it takes before a signal is observed. A better performance measure for VSI charts would be the ATS or the AATS.

The values obtained for $W^{+}$are discrete. Recall that $U C L=c$ and $L C L=\frac{n(n+1)}{2}-c$, with $c$ chosen for a specified nominal $A R L_{0}$. Taking this into account we can now partition our control chart into regions corresponding to the two time intervals. Following the procedure in Amin and Widmaier ${ }^{5}$ partition the range of discrete values $\left(\frac{n(n+1)}{2}-c, \ldots, c\right)$ into two regions, $I_{1}$ and $I_{2}$, where $I_{1}=\left\{\frac{n(n+1)}{2}-c, \ldots, \frac{n(n+1)}{2}-k-1\right\} \cup\{k+1, \ldots, c\}$ and $I_{2}=\left\{\frac{n(n+1)}{2}-k, \ldots, k\right\}$. Following the idea in Amin and Widmaier ${ }^{5}$, the chart will signal in the regions $\{0, \ldots, n-c-1\} \cup\{c+1, \ldots, n\}$. The regions defined as $I_{1}$ and $I_{2}$ correspond to the two time intervals. This means that if $W^{+} \in I_{1}$, wait $d_{1}$ time units until sampling the next sample and if $W^{+} \in I_{2}$, wait $d_{2}$ time units until sampling the next sample, where $d_{1}<d_{2}$.

The probabilities corresponding to the different regions of the chart are given by

$$
\begin{gathered}
p_{j}=\operatorname{Pr}\left(W^{+} \in I_{j}\right), \\
p_{0 j}=\operatorname{Pr}\left(W^{+} \in I_{j} \mid I C\right), \\
p_{1 j}=\operatorname{Pr}\left(W^{+} \in I_{j} \mid O O C\right)
\end{gathered}
$$

where $j=1,2$. 


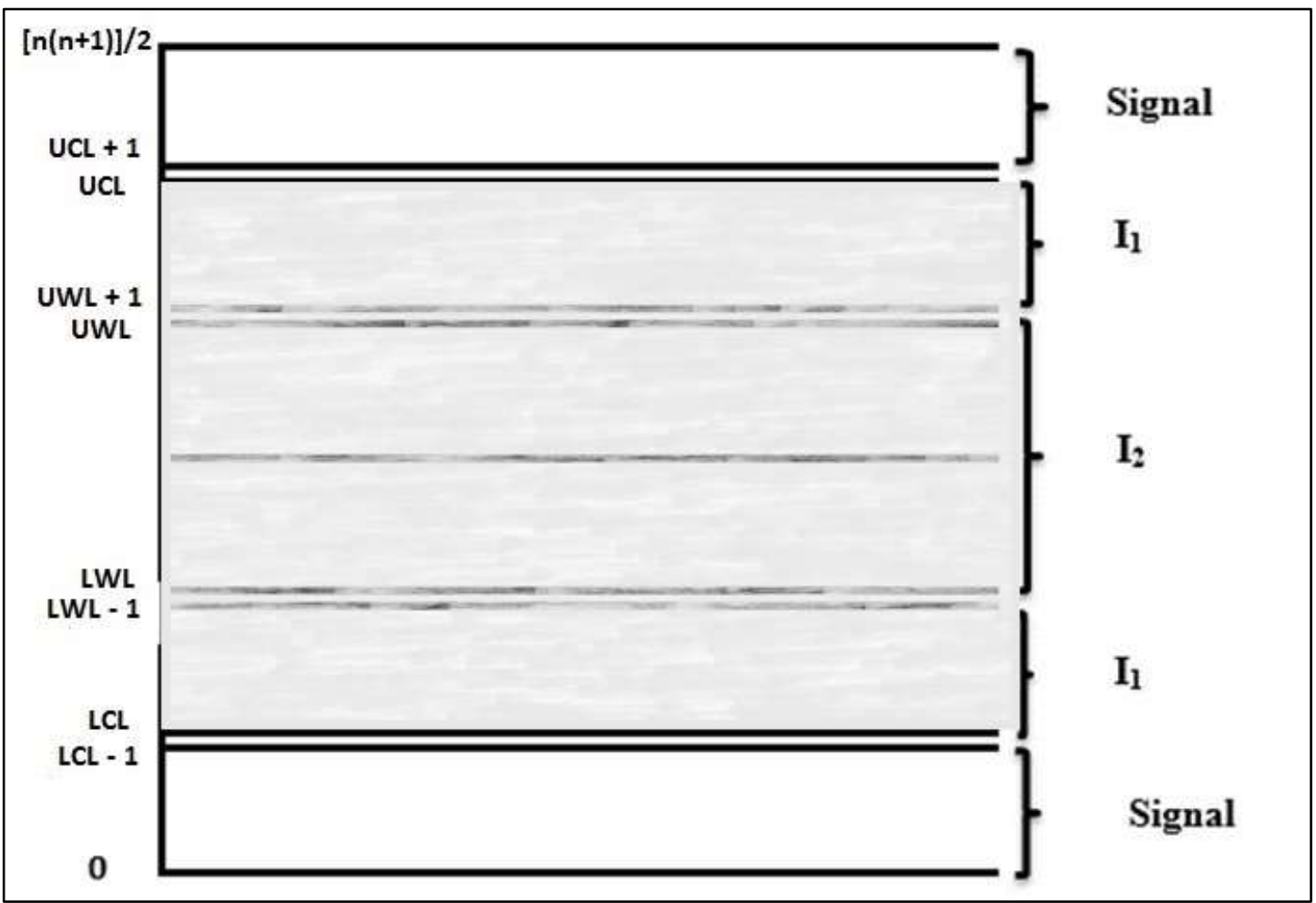

Figure 3. The regions of a VSI signed-rank control chart, where $\frac{n(n+1)}{2}=$ the maximum value attainable by the charting statistic, $c=U C L, k=U W L, \frac{n(n+1)}{2}-k=k^{\prime}=L W L, \frac{n(n+1)}{2}-c=L C L$. If the charting statistic plots in the grey region, then the process is IC, otherwise it is deemed OOC

The probabilities of observing a signal in the IC and OOC cases are given by

$$
\alpha_{0}=\operatorname{Pr}\left(\left(W^{+}<\frac{n(n+1)}{2}-c\right) \cup\left(W^{+}>c\right) \mid \theta=\theta_{0}\right)
$$

and

$$
\alpha_{1}=\operatorname{Pr}\left(\left(W^{+}<n-c\right) \cup\left(W^{+}>c\right) \mid \theta=\theta_{1}\right)
$$

respectively. Therefore,

$$
\begin{gathered}
p_{01}=\operatorname{Pr}\left(\left(\frac{n(n+1)}{2}-c<W^{+}<\frac{n(n+1)}{2}-k-1\right) \cup\left(k+1<W^{+}<c\right) \mid \theta=\theta_{0}\right) \\
p_{02}=\operatorname{Pr}\left(\frac{n(n+1)}{2}-k<W^{+}<k \mid \theta=\theta_{0}\right) \\
p_{11}=\operatorname{Pr}\left(\left(\frac{n(n+1)}{2}-c<W^{+}<\frac{n(n+1)}{2}-k-1\right) \cup\left(k+1<W^{+}<c\right) \mid \theta=\theta_{1}\right) \\
p_{12}=\operatorname{Pr}\left(\frac{n(n+1)}{2}-k<W^{+}<k \mid \theta=\theta_{1}\right)
\end{gathered}
$$


The value of $k$ has to be chosen such that it lies within the $C L$ and the upper control limit (c). In choosing the value of $k$ one has to take into account what the values of $d_{1}$ and $d_{2}$ should be. Following the suggestions in Reynolds et al. ${ }^{1}$, Amin and Widmaier ${ }^{5}$ and other related articles, if $d=$ the fixed sampling interval, then the value of $d_{1}$, which is measured in units of $d$ should be specified and it should be as small as is practical (e.g. $0.1 d$ ). The value of $d_{2}$, also measured in units of $d$, is then calculated using the formula $d_{2}=\left(1-\alpha_{0}-d_{1} p_{01}\right) / p_{02}$. It has been recommended by Reynolds et al. ${ }^{1}$ and others following that article, that $1.5 d \leq d_{2} \leq 2 d$. For example, if the fixed time interval is $d=60$ minutes, then the time interval $d_{1}$, could be taken as small as $0.1 d$, which would make that interval $d_{1}=0.1 \times 60=6$ minutes. Depending on where $k$ would lie, $d_{2}$ would then be calculated. It should lie within the interval mentioned above. If it was determined to be $1.5 d$, then the longer time interval $d_{2}=1.5 \times 60=90$ minutes.

The IC probabilities of the signed-rank chart can easily be obtained from the Wilcoxon signed-rank probability distribution tables. However, the OOC distribution of the Wilcoxon signed-rank statistic is difficult to obtain (Gibbons and Chakraborti ${ }^{17}$ page 205). This means that the OOC probabilities $\left(p_{11}, p_{12}\right.$ and $\left.\alpha_{1}\right)$ for the VSI control chart, which are needed for the calculation of the AATS, are also very difficult to obtain analytically. For this reason, we used extensive Monte Carlo simulations with 100,000 iterations, using $\operatorname{SAS}^{\circledR} \mathrm{v}$ 9.3, to find the OOC probabilities. The values for the IC probabilities were compared to available Wilcoxon signed-rank probability tables. In order to have the VSI $\bar{X}$-chart and the VSI sign chart on equal footing, we also used Monte Carlo simulations for those charts.

Step 1: After specifying the sample size, calculate the control limits and warning limits according to a specified IC AATS and the value needed for the time interval $d_{2}$.

Step 2: Generate a random sample from some process distribution, say, the normal distribution.

Step 3: Calculate the charting statistic for the sample and compare it to the control limits and warning limits calculated in Step 1.

Step 4: Run a counter for each region, $I_{1}, I_{2}$ and the signalling area. Increase the counter by 1 for the area into which the charting statistic plots.

Step 5: $\quad$ Repeat Steps 1 to 4 a total of 100,000 times. 
Step 6: For the VSI probabilities $\left(p_{01}, p_{02}, p_{11}, p_{12}, \alpha_{0}\right.$ and $\left.\alpha_{1}\right)$, once we have obtained a 'dataset' with 100,000 observations we take the counters for each region and divide each counter by 100,000 to obtain the probabilities.

\section{Performance comparisons}

For the performance comparisons we considered four underlying process distributions, the standard normal $(N(0,1))$, the Uniform $(0,1)$, the Laplace $(0,1)$ and the $t$-distribution with 3 degrees of freedom $(t(3))$. These distributions are all symmetric distributions as the signedrank statistic assumes a symmetric process distribution. All three control charts were designed to have the same $A R L_{0}$ and approximately the same time interval units. We specified $d_{1}=0.1$ and using this we found warning limits which would yield the second time interval to be as close as possible: to $d_{2}=1.5$. Due to the discreteness of the nonparametric charts, it was not possible to have values for $d_{2}$ exactly at 1.5 . The warning limits, $k$ and $k^{\prime}$, were also found using Monte Carlo simulations to have the longer time interval $d_{2} \approx 1.5$. In the IC case the ARL and the AATS should have the same value. Then shifts were added to the process mean. Since we only considered symmetrical distributions, we only considered positive shifts in the process mean, as the results would be the same for the negative shifts due to the symmetry. Shifts of size $\delta=0.1,0.15,0.2,0.25(0.25) 3$ were considered. These shifts are in units of standard deviations of the original observations. Very small shifts were chosen, since the charts all converged very quickly to approximately 0.7 for larger shifts and it was not clear in some cases whether there was a difference in the performance between the VSI signed-rank chart and the VSI sign chart.

First we will show a comparison of the newly proposed VSI signed-rank chart with its FSI counterpart. The performance measure used for this comparison will be the AATS. This comparison will show that the proposed VSI signed-rank chart outperforms the FSI signedrank chart.

Following this, a comparison of the FSI $\bar{X}$ - chart, the FSI sign chart and the FSI signedrank chart (using $W^{+}$as charting statistic), with the $A R L$ as a measure of performance, will be shown. This is done to show how the traditional control charts perform in comparison to each other.

The VSI $\bar{X}$-chart probability calculations were also done using Monte Carlo simulations. The steps followed are the same as those for the VSI signed-rank chart, as shown earlier, the only difference is the calculation of the charting statistic. The values of the design constant and the warning limits were adjusted in order to find the desired $A R L_{0}$ and the desired IC 
probabilities $\left(p_{01}\right.$ and $\left.p_{02}\right)$. Lastly we will show a comparison of the VSI $\bar{X}$-chart, the VSI sign chart and the VSI signed-rank chart. The purpose of this is to show how well the newly proposed VSI signed-rank chart performs compared to the two other charts. Since the FSI and VSI $\bar{X}$-charts are parametric charts, based on the assumption of normality, it is expected that these charts will outperform the two nonparametric charts when the underlying process distribution is normal. However, when the underlying process distribution is non-normal, we expect the nonparametric charts to perform better.

In doing the comparisons, we started off using a sample size $n=10$. We quickly realised that the results of the VSI sign chart and the VSI signed-rank chart converged very quickly (they reach a value which stays constant) making it difficult to justify how much better the one chart was performing over the other chart. For this reason we will show results for a larer sample size of $n=30$, where the results are clearer.

For the VSI $\bar{X}$-chart the values of $r$, the design constant for the control limits, as well as the values of $r^{\prime}$, the design constant for the warning limits, had to be adjusted for each distribution, in order to have an IC AATS and IC ARL that is the same for all charts. These adjusted values can be found in Table 1. Because the nonparametric charts have the same IC run-length distribution over all continuous distributions, the control limits did not have to be adjusted for the different distributions.

Table 1. Upper control limits, upper warning limits and design constants for $n=30$ and IC AATS $=700$.

\begin{tabular}{|l|c|c|c|c|c|c|}
\hline Distribution & \multicolumn{2}{|c|}{ VSI signed-rank chart } & \multicolumn{2}{c|}{ VSI sign chart } & \multicolumn{2}{c|}{ VSI $\overline{\boldsymbol{X}}$-chart } \\
\hline & $\boldsymbol{U} \boldsymbol{C} \boldsymbol{L}$ & $\boldsymbol{U W L}$ & $\boldsymbol{U} \boldsymbol{L}$ & $\boldsymbol{U} \boldsymbol{L}$ & $\boldsymbol{r}$ & $\boldsymbol{r}^{\prime}$ \\
\hline $\boldsymbol{N}(\mathbf{0 , 1})$ & 381 & 277 & 23 & 17 & 3.098 & 0.915 \\
\hline Uniform & 381 & 277 & 23 & 17 & 3.591 & 2.86 \\
\hline Laplace & 381 & 277 & 23 & 17 & 3.178 & 0.955 \\
\hline $\boldsymbol{t}(\mathbf{3})$ & 381 & 277 & 23 & 17 & 4.341 & 0.888 \\
\hline
\end{tabular}

Table 1 shows the upper control limits and upper warning limits for the VSI signed-rank chart and the VSI sign chart, as well as the design constants $r$ and $r$, for the VSI $\bar{X}$ - chart with $n=30$.

\subsection{VSI signed-rank vs FSI signed-rank chart}

In Table 2 we show the AATS for the FSI signed-rank control chart and the VSI signed-rank control chart for the four distributions under consideration for $n=30$. 
Table 2. AATS of FSI and VSI signed-rank control chart for location for $n=30$.

\begin{tabular}{|c|c|c|c|c|c|c|c|c|}
\hline Shift & \multicolumn{2}{|c|}{$N(0,1)$} & \multicolumn{2}{c|}{ Uniform $(0,1)$} & \multicolumn{2}{c|}{ Laplace $(0,1)$} & \multicolumn{2}{c|}{$t(3)$} \\
\hline$\delta$ & FSI & VSI & FSI & VSI & FSI & VSI & FSI & VSI \\
\hline 0.00 & 700 & 700 & 700 & 700 & 700 & 700 & 700 & 700 \\
\hline 0.10 & 270.72 & 247.41 & 298.72 & 272.36 & 171.29 & 148.76 & 143.97 & 120.45 \\
\hline 0.15 & 135.37 & 110.73 & 163.33 & 133.81 & 73.94 & 54.20 & 56.18 & 38.07 \\
\hline 0.20 & 70.52 & 49.58 & 89.85 & 63.90 & 35.55 & 21.19 & 25.45 & 13.35 \\
\hline 0.25 & 37.90 & 22.23 & 49.41 & 29.70 & 18.54 & 8.85 & 12.60 & 5.17 \\
\hline 0.50 & 3.73 & 1.30 & 5.02 & 1.63 & 2.13 & 0.95 & 1.35 & 0.83 \\
\hline 0.75 & 0.94 & 0.77 & 1.17 & 0.80 & 0.76 & 0.75 & 0.60 & 0.74 \\
\hline 1.00 & 0.54 & 0.73 & 0.57 & 0.73 & 0.54 & 0.73 & 0.51 & 0.73 \\
\hline 1.25 & 0.50 & 0.73 & 0.50 & 0.73 & 0.50 & 0.73 & 0.50 & 0.73 \\
\hline 1.50 & 0.50 & 0.73 & 0.50 & 0.73 & 0.50 & 0.73 & 0.50 & 0.73 \\
\hline 1.75 & 0.50 & 0.73 & 0.50 & 0.73 & 0.50 & 0.73 & 0.50 & 0.73 \\
\hline 2.00 & 0.50 & 0.73 & 0.50 & 0.73 & 0.50 & 0.73 & 0.50 & 0.73 \\
\hline 2.25 & 0.50 & 0.73 & 0.50 & 0.73 & 0.50 & 0.73 & 0.50 & 0.73 \\
\hline 2.50 & 0.50 & 0.73 & 0.50 & 0.73 & 0.50 & 0.73 & 0.50 & 0.73 \\
\hline 2.75 & 0.50 & 0.73 & 0.50 & 0.73 & 0.50 & 0.73 & 0.50 & 0.73 \\
\hline 3.00 & 0.50 & 0.73 & 0.50 & 0.73 & 0.50 & 0.73 & 0.50 & 0.73 \\
\hline
\end{tabular}

In Table 2, and in the upcoming tables, chart that performs the best is highlighted in grey. It can be seen that the VSI signed-rank chart outperforms the FSI counterpart. When $n=30$, the VSI chart also performs better than the FSI chart for small shifts. Since the FSI chart still converges to smaller AATS values, this means that the VSI chart performs better only for very small shifts, i.e. 0.1 to 0.75 . Since shifts are quite often smaller rather than large, this speaks in favour of the VSI chart, which clearly performs better in such a case.

\subsection{FSI chart comparisons}

In Tables 3 to 6 we show the comparison of the FSI signed-rank chart, the FSI sign chart and the FSI $\bar{X}$-chart all with sample size $n=30$, with the $A R L$ as performance measure. This comparison is shown, in order to establish which original chart performed better in which situation. 
Table 3. $A R L$ values of the FSI signed-rank, FSI sign and FSI $\bar{X}$-charts for $n=30$

\begin{tabular}{|c|c|c|c|}
\hline Shift & \multicolumn{3}{|c|}{ Data from $\boldsymbol{N}(\mathbf{0 , 1})$} \\
\hline$\delta$ & FSI signed-rank & FSI sign & FSI $\overline{\boldsymbol{X}}$ - chart \\
\hline 0.00 & 700 & 700 & 700 \\
\hline 0.10 & 271.22 & 331.18 & 235.63 \\
\hline 0.15 & 135.87 & 183.73 & 110.30 \\
\hline 0.20 & 71.02 & 103.33 & 54.70 \\
\hline 0.25 & 38.40 & 60.22 & 28.84 \\
\hline 0.50 & 4.23 & 7.32 & 3.06 \\
\hline 0.75 & 1.44 & 2.15 & 1.22 \\
\hline 1.00 & 1.04 & 1.23 & 1.01 \\
\hline 1.25 & 1.00 & 1.03 & 1.00 \\
\hline 1.50 & 1.00 & 1.00 & 1.00 \\
\hline 1.75 & 1.00 & 1.00 & 1.00 \\
\hline 2.00 & 1.00 & 1.00 & 1.00 \\
\hline 2.25 & 1.00 & 1.00 & 1.00 \\
\hline 2.50 & 1.00 & 1.00 & 1.00 \\
\hline 2.75 & 1.00 & 1.00 & 1.00 \\
\hline 3.00 & 1.00 & 1.00 & 1.00 \\
\hline
\end{tabular}

Table 4. $A R L$ values of the FSI signed-rank, FSI sign and FSI $\bar{X}$-charts for $n=30$

\begin{tabular}{|c|c|c|c|}
\hline Shift & \multicolumn{3}{|c|}{ Data from Uniform(0,1) } \\
\hline$\delta$ & FSI signed-rank & FSI sign & FSI $\overline{\boldsymbol{X}}$ - chart \\
\hline 0.00 & 700 & 700 & 700 \\
\hline 0.10 & 299.22 & 445.04 & 128.17 \\
\hline 0.15 & 163.83 & 304.97 & 61.46 \\
\hline 0.20 & 90.35 & 197.39 & 31.85 \\
\hline 0.25 & 49.91 & 125.68 & 17.59 \\
\hline 0.50 & 5.52 & 19.30 & 2.41 \\
\hline 0.75 & 1.67 & 4.73 & 1.14 \\
\hline 1.00 & 1.07 & 1.83 & 1.01 \\
\hline 1.25 & 1.00 & 1.13 & 1.00 \\
\hline 1.50 & 1.00 & 1.00 & 1.00 \\
\hline 1.75 & 1.00 & 1.00 & 1.00 \\
\hline 2.00 & 1.00 & 1.00 & 1.00 \\
\hline 2.25 & 1.00 & 1.00 & 1.00 \\
\hline 2.50 & 1.00 & 1.00 & 1.00 \\
\hline 2.75 & 1.00 & 1.00 & 1.00 \\
\hline 3.00 & 1.00 & 1.00 & 1.00 \\
\hline
\end{tabular}


Table 5. $A R L$ values of the FSI signed-rank, FSI sign and FSI $\bar{X}$-charts for $n=30$

\begin{tabular}{|c|c|c|c|}
\hline Shift & \multicolumn{3}{|c|}{ Data from Laplace(0,1) } \\
\hline$\delta$ & FSI signed-rank & FSI sign & FSI $\overline{\boldsymbol{X}}$ - chart \\
\hline 0.00 & 700 & 700 & 700 \\
\hline 0.10 & 171.79 & 151.29 & 276.09 \\
\hline 0.15 & 74.44 & 65.01 & 131.41 \\
\hline 0.20 & 36.05 & 31.87 & 65.36 \\
\hline 0.25 & 19.04 & 17.59 & 34.95 \\
\hline 0.50 & 2.63 & 2.75 & 3.42 \\
\hline 0.75 & 1.26 & 1.34 & 1.25 \\
\hline 1.00 & 1.04 & 1.07 & 1.01 \\
\hline 1.25 & 1.00 & 1.01 & 1.00 \\
\hline 1.50 & 1.00 & 1.00 & 1.00 \\
\hline 1.75 & 1.00 & 1.00 & 1.00 \\
\hline 2.00 & 1.00 & 1.00 & 1.00 \\
\hline 2.25 & 1.00 & 1.00 & 1.00 \\
\hline 2.50 & 1.00 & 1.00 & 1.00 \\
\hline 2.75 & 1.00 & 1.00 & 1.00 \\
\hline 3.00 & 1.00 & 1.00 & 1.00 \\
\hline
\end{tabular}

Table 6. $A R L$ values of the FSI signed-rank, FSI sign and FSI $\bar{X}$-charts for $n=30$

\begin{tabular}{|c|c|c|c|}
\hline Shift & \multicolumn{3}{|c|}{ Data from $\boldsymbol{t}(\mathbf{3})$} \\
\hline$\delta$ & FSI signed-rank & FSI sign & FSI $\overline{\boldsymbol{X}}$ - chart \\
\hline 0.00 & 700 & 700 & 700 \\
\hline 0.10 & 144.47 & 164.61 & 605.33 \\
\hline 0.15 & 56.68 & 68.56 & 494.80 \\
\hline 0.20 & 25.95 & 31.35 & 371.20 \\
\hline 0.25 & 13.10 & 16.14 & 258.73 \\
\hline 0.50 & 1.85 & 2.11 & 20.92 \\
\hline 0.75 & 1.10 & 1.14 & 2.51 \\
\hline 1.00 & 1.01 & 1.02 & 1.13 \\
\hline 1.25 & 1.00 & 1.00 & 1.01 \\
\hline 1.50 & 1.00 & 1.00 & 1.00 \\
\hline 1.75 & 1.00 & 1.00 & 1.00 \\
\hline 2.00 & 1.00 & 1.00 & 1.00 \\
\hline 2.25 & 1.00 & 1.00 & 1.00 \\
\hline 2.50 & 1.00 & 1.00 & 1.00 \\
\hline 2.75 & 1.00 & 1.00 & 1.00 \\
\hline 3.00 & 1.00 & 1.00 & 1.00 \\
\hline
\end{tabular}


The FSI $\bar{X}$-chart is still expected to perform better than the two nonparametric charts if the underlying process distribution is $N(0,1)$. This is also the case as can be seen in the Table 3 below. The $\bar{X}$-chart performs much better than the nonparametric charts for small shift of size 0.1 to 1 . The charts all converge sooner when the sample size is larger and they all converge to 1 for shifts larger than 1 .

When the underlying distribution is Uniform $(0,1)$, the FSI $\bar{X}$-chart still outperforms the FSI signed-rank and FSI sign chart by quite a margin for small shifts up to a shift of size 1 . The FSI signed-rank chart however still performs much better than the sign chart for small shifts. For shift greater than 1 , all three charts converge to an $A R L_{\delta}$ of 1.

When the underlying distribution is Laplace $(0,1)$, the FSI sign chart, now with the larger sample size, clearly performs best for small shifts of size 0.1 to 0.25 . This is expected (as it can be seen from Gibbons and Chakraborti ${ }^{17}$ page 492) that the Asymptotic Relative Efficiency (ARE) of the sign test relative to the signed-rank test is $4 / 3$ for the Laplace distribution. For shifts greater than 1 the charts all converge to an $A R L_{\delta}$ of 1 again.

When the underlying distribution is $t(3)$, the FSI signed-rank chart performs better than the other two distributions for small shifts. The FSI sign chart does not perform much worse, however the FSI $\bar{X}$-chart performs much worse. For shifts larger than 1.5 all three charts converge to 1 .

\subsection{VSI Chart comparisons}

In Tables 7 to 10 we show the comparison of the VSI signed-rank chart, the VSI sign chart and the VSI $\bar{X}$ - chart in terms of their AATS values.

It is expected that the performance of the charts will be very similar to the corresponding FSI charts in relation with each other, since in principle each of the charts did not change much other than the inclusion of the time intervals for sampling. We do, however, already know that all the VSI charts perform better than their FSI counterparts, in this regard see the comparison of the FSI signed-rank and the VSI signed-rank charts in Table 2 and see Amin and Widmaier ${ }^{5}$ and Reynolds et al. ${ }^{1}$ for the performance of the VSI sign chart and the VSI $\bar{X}$ chart, respectively. 
Table 7. AATS values of the VSI signed-rank, VSI sign and VSI $\bar{X}$-charts for $n=30$

\begin{tabular}{|c|c|c|c|}
\hline Shift & \multicolumn{3}{|c|}{ Data from $N(\mathbf{0 , 1})$} \\
\hline$\delta$ & VSI signed-rank & VSI sign & VSI $\overline{\boldsymbol{X}}$ - chart \\
\hline 0.00 & 700 & 700 & 700 \\
\hline 0.10 & 247.41 & 310.60 & 213.94 \\
\hline 0.15 & 110.73 & 159.28 & 88.59 \\
\hline 0.20 & 49.58 & 80.43 & 37.35 \\
\hline 0.25 & 22.23 & 41.01 & 16.24 \\
\hline 0.50 & 1.30 & 2.40 & 1.08 \\
\hline 0.75 & 0.77 & 0.87 & 0.75 \\
\hline 1.00 & 0.73 & 0.75 & 0.73 \\
\hline 1.25 & 0.73 & 0.73 & 0.73 \\
\hline 1.50 & 0.73 & 0.73 & 0.73 \\
\hline 1.75 & 0.73 & 0.73 & 0.73 \\
\hline 2.00 & 0.73 & 0.73 & 0.73 \\
\hline 2.25 & 0.73 & 0.73 & 0.73 \\
\hline 2.50 & 0.73 & 0.73 & 0.73 \\
\hline 2.75 & 0.73 & 0.73 & 0.73 \\
\hline 3.00 & 0.73 & 0.73 & 0.73 \\
\hline
\end{tabular}

Table 8. AATS values of the VSI signed-rank, VSI sign and VSI $\bar{X}$-charts for $n=30$

\begin{tabular}{|c|c|c|c|}
\hline Shift & \multicolumn{3}{|c|}{ Data from Uniform(0,1) } \\
\hline$\delta$ & VSI signed-rank & VSI sign & VSI $\overline{\boldsymbol{X}}$ - chart \\
\hline 0.00 & 700 & 700 & 700 \\
\hline 0.10 & 272.36 & 430.32 & 94.52 \\
\hline 0.15 & 133.81 & 282.85 & 36.60 \\
\hline 0.20 & 63.90 & 172.82 & 15.07 \\
\hline 0.25 & 29.70 & 102.03 & 6.64 \\
\hline 0.50 & 1.63 & 8.93 & 0.91 \\
\hline 0.75 & 0.80 & 1.48 & 0.75 \\
\hline 1.00 & 0.73 & 0.83 & 0.73 \\
\hline 1.25 & 0.73 & 0.74 & 0.73 \\
\hline 1.50 & 0.73 & 0.73 & 0.73 \\
\hline 1.75 & 0.73 & 0.73 & 0.73 \\
\hline 2.00 & 0.73 & 0.73 & 0.73 \\
\hline 2.25 & 0.73 & 0.73 & 0.73 \\
\hline 2.50 & 0.73 & 0.73 & 0.73 \\
\hline 2.75 & 0.73 & 0.73 & 0.73 \\
\hline 3.00 & 0.73 & 0.73 & 0.73 \\
\hline
\end{tabular}


Table 9. AATS values of the VSI signed-rank, VSI sign and VSI $\bar{X}$ - charts for $n=30$

\begin{tabular}{|c|c|c|c|}
\hline Shift & \multicolumn{3}{|c|}{ Data from Laplace(0,1) } \\
\hline$\delta$ & VSI signed-rank & VSI sign & VSI $\overline{\boldsymbol{X}}$ - chart \\
\hline 0.00 & 700 & 700 & 700 \\
\hline 0.10 & 148.76 & 127.10 & 258.24 \\
\hline 0.15 & 54.20 & 45.32 & 108.84 \\
\hline 0.20 & 21.19 & 17.79 & 45.91 \\
\hline 0.25 & 8.85 & 7.82 & 20.16 \\
\hline 0.50 & 0.95 & 0.98 & 1.15 \\
\hline 0.75 & 0.75 & 0.77 & 0.76 \\
\hline 1.00 & 0.73 & 0.74 & 0.73 \\
\hline 1.25 & 0.73 & 0.73 & 0.73 \\
\hline 1.50 & 0.73 & 0.73 & 0.73 \\
\hline 1.75 & 0.73 & 0.73 & 0.73 \\
\hline 2.00 & 0.73 & 0.73 & 0.73 \\
\hline 2.25 & 0.73 & 0.73 & 0.73 \\
\hline 2.50 & 0.73 & 0.73 & 0.73 \\
\hline 2.75 & 0.73 & 0.73 & 0.73 \\
\hline 3.00 & 0.73 & 0.73 & 0.73 \\
\hline
\end{tabular}

Table 10. AATS values of the VSI signed-rank, VSI sign and VSI $\bar{X}$ - charts for $n=30$

\begin{tabular}{|c|c|c|c|}
\hline Shift & \multicolumn{3}{|c|}{ Data from $\boldsymbol{t}(\mathbf{3})$} \\
\hline$\delta$ & VSI signed-rank & VSI sign & VSI $\overline{\boldsymbol{X}}$ - chart \\
\hline 0.00 & 700 & 700 & 700 \\
\hline 0.10 & 120.45 & 140.26 & 554.90 \\
\hline 0.15 & 38.07 & 48.26 & 391.74 \\
\hline 0.20 & 13.35 & 17.36 & 239.92 \\
\hline 0.25 & 5.17 & 6.95 & 130.64 \\
\hline 0.50 & 0.83 & 0.87 & 3.55 \\
\hline 0.75 & 0.74 & 0.74 & 0.89 \\
\hline 1.00 & 0.73 & 0.73 & 0.74 \\
\hline 1.25 & 0.73 & 0.73 & 0.73 \\
\hline 1.50 & 0.73 & 0.73 & 0.73 \\
\hline 1.75 & 0.73 & 0.73 & 0.73 \\
\hline 2.00 & 0.73 & 0.73 & 0.73 \\
\hline 2.25 & 0.73 & 0.73 & 0.73 \\
\hline 2.50 & 0.73 & 0.73 & 0.73 \\
\hline 2.75 & 0.73 & 0.73 & 0.73 \\
\hline 3.00 & 0.73 & 0.73 & 0.73 \\
\hline
\end{tabular}

It can be seen that the VSI $\bar{X}$-chart performs better when the underlying process distribution is $N(0,1)$ with the VSI signed-rank chart still outperforming the VSI sign chart. For the Uniform $(0,1)$ distribution the VSI $\bar{X}$-chart performs much better than the other two charts, with the VSI sign chart performing the worst. The VSI signed-rank chart performs much better than the VSI sign chart, but still worse than the VSI $\bar{X}$-chart. For the 
Laplace $(0,1)$ distribution the VSI sign chart performs best for small shifts of 0.1 to 0.5 , which again is expected. The VSI signed-rank chart performs slightly worse than the VSI sign chart, however the VSI $\bar{X}$-chart performs the worst. For the $t(3)$ distribution the VSI signed-rank chart performs the best. The VSI sign chart performs slightly worse and the VSI $\bar{X}$-chart performs very much worse than both the nonparametric charts. For large shifts, for all four distributions, the charts all converge to approximately 0.73 .

Table 11. Summary of chart performances

\begin{tabular}{|l|c|c|c|c|}
\hline & \multicolumn{4}{|c|}{ Distribution and rank of charts } \\
\hline Chart & $\mathbf{N}(\mathbf{0 , 1})$ & Uniform(0,1) & Laplace(0,1) & $\mathbf{t}(\mathbf{3})$ \\
\hline VSI signed-rank & 2 & 2 & 2 & 1 \\
\hline VSI sign & 3 & 3 & 1 & 2 \\
\hline VSI $\bar{X}$ - chart & 1 & 1 & 3 & 3 \\
\hline
\end{tabular}

From Table 11 it can be seen that the VSI signed-rank chart performs best when the underlying process distribution is symmetric by heavier-tailed than the normal distribution.

\section{Summary and conclusions}

We propose a new nonparametric chart under the variable sampling interval scheme. This is the VSI signed-rank control chart. The chart was expected to perform better than the FSI signed-rank chart and also to perform better or similar to the nonparametric VSI sign chart proposed by Amin and Widmaier ${ }^{5}$, since the signed-rank test is a more powerful test than the sign test. In the comparisons we found that the VSI signed-rank chart does indeed perform better than the FSI signed-rank chart for the $N(0,1)$, Uniform $(0,1)$, Laplace $(0,1)$ and the $t(3)$ distributions. We only used symmetric distributions, as the signed-rank test assumes symmetry. We also found that the VSI signed-rank chart performs better than the VSI sign chart for the $N(0,1)$, the Uniform $(0,1)$ and the $t(3)$ distribution. Only for the Laplace $(0,1)$ distribution did the VSI sign chart perform better than the VSI signed-rank chart, which can be expected, since the asymptotic relative efficiency of the sign test relative to the signedrank test is $4 / 3$. We included the parametric VSI $\bar{X}$-chart proposed by Reynolds et al. ${ }^{1}$ in our comparison as a benchmark. This chart was expected to perform better when the normality assumption is true, which is seen to be the case. The VSI $\bar{X}$ - chart also performed better than the VSI signed-rank chart when the process follows a Uniform $(0,1)$ distribution. However, for the Laplace $(0,1)$ and the $\mathrm{t}(3)$ distributions, the VSI $\bar{X}$-chart performed considerably worse than the VSI signed-rank and the VSI sign chart. These results speak in favour of using the 
proposed nonparametric VSI signed-rank chart when the data follow an unknown symmetric distribution. It also shows the importance of nonparametric control charts in statistical process control.

\section{Acknowledgements}

This research was supported in part by the South African Research Chairs Initiative at the University of Pretoria and by the Department of Information Systems, Statistics and Management Science, University of Alabama. Marien Graham's research was also supported by the National Research Foundation (Thuthuka program: TTK14061168807; grant number: 94102).

\section{References}

1. Reynolds, MR, Amin, RW, Arnold, JC, Nachlas, JA. $\bar{X}$ Charts with Variable Sampling Intervals. Technometrics 1988; 30(2), 181-192.

2. Reynolds, MR, Amin, RW, Arnold, JC. CUSUM charts with variable sampling intervals. Technometrics 1990; 32, 371-384.

3. Rendtel, U. CUSUM Schemes with Variable Sampling Intervals and Sampling Size. Statistical Papers 1990; 31, 103-118.

4. Saccucci, MS, Amin, RW, Lucas, JM. Exponentially Weighted Moving Average Control Schemes with Variable Sampling Intervals. Communications in Statistics: Simulation and Computation 1992; 21, 627-657.

5. Amin, RW, Widmaier, O. Sign Control Charts with Variable Sampling Intervals. Communications in Statistics: Theory and Methods 1999; 28, 1961-1985.

6. Reynolds, MR, Arnold, JC. Optimal Shewhart Control Charts with Variable Sampling Intervals between Samples. Sequential Analysis 1989; 8, 51-77.

7. Jensen, A.W, Bryce, GR, Reynolds, MR. Design Issues for Adaptive Control Charts. Quality and Reliability Engineering International 2008; 24, 429 - 445.

8. Epprecht, EK, Simoes, BFT, Mendes, FCT. A variable sampling interval EWMA chart for attributes. International Journal of Advanced Manufacturing Technology 2010; 49, $281-292$.

9. Ou, Y, Wu, A, Yu, FJ, Shamsuzzaman, M. An SPRT control chart with variable sampling intervals. International Journal of Advanced Manufacturing Technology 2011; 56(9-12), 1149 - 1158.

10. Zhang, Y, Castagliola, P, Wu. Z, Khoo, MBC. The Variable Sampling Interval $\bar{X}$ Chart with Estimated Parameters. Quality and Reliability Engineering International 2012; 28, $19-34$.

11. Reynolds, MR. Optimal Variable Sampling Interval Control Charts. Sequential Analysis 1989; 8, 361-379.

12. Runger, GC, Pignatiello, JJ. Adaptive Sampling for Process Control. Journal of Quality Technology 1991; 23, 135-155.

13. Reynolds, MR. Evaluating Properties of Variable Sampling Interval Control Charts. Sequential Analysis; 1995, 14, 59-97. 
14. Chakraborti, S, Van der Laan, P, Bakir, ST. "Nonparametric control charts: An overview and some results." Journal of Quality Technology 2001; 33 (3), 304-315.

15. Chakraborti, S, Graham, MA. "Nonparametric control charts." Encyclopedia of Statistics in Quality and Reliability 2007; 1, 415 - 429, John Wiley, New York.

16. Chakraborti, S, Human, SW, Graham, MA. "Nonparametric (distribution-free) quality control charts." In Handbook of Methods and Applications of Statistics: Engineering, Quality Control, and Physical Sciences. N. Balakrishnan, Ed. 2011; 298-329, John Wiley \& Sons, New York.

17. Gibbons, JD, Chakraborti, S. Nonparametric Statistical Inference, 5th ed. 2010, Taylor and Francis, Boca Raton, FL.

18. Bakir, ST. A distribution-free Shewhart quality control chart based on signed-ranks. Quality Engineering 2004; 16, 613-623.

19. Human, SW, Graham, MA. Average run lengths and operating characteristic curves." Encyclopedia of Statistics in Quality and Reliability 2007; 1, 159-168, John Wiley, New York. 